\title{
Effects of quark matter nucleation on the evolution of proto-neutron stars
}

\author{
I. Bombaci ${ }^{1,2}$, D. Logoteta ${ }^{3}$, C. Providência ${ }^{3}$, and I. Vidaña ${ }^{3}$ \\ 1 Dipartimento di Fisica “E. Fermi”, Università di Pisa, Largo B. Pontecorvo 3, 56127 Pisa, Italy \\ e-mail: bombaci@df.unipi.it \\ 2 INFN, Sezione di Pisa, Largo B. Pontecorvo 3, 56127 Pisa, Italy \\ ${ }^{3}$ Centro de Física Computacional, Department of Physics, University of Coimbra, 3004-516 Coimbra, Portugal
}

Received 18 September 2010 / Accepted 3 December 2010

\begin{abstract}
Context. A phase of strong interacting matter with deconfined quarks is expected in the core of a massive neutron star. If this deconfinement phase transition is of the first order, as suggested by many models inspired by quantum chromodynamics, then it will be triggered by the nucleation of a critical size drop of the (stable) quark phase in the metastable hadronic phase. Within these circumstances it has been shown that cold $(T=0)$ pure hadronic compact stars above a threshold value of their gravitational mass (central pressure) are metastable with respect to the "decay" (conversion) to quark stars (i.e., compact stars made at least in part of quark matter). This stellar conversion process liberates a huge amount of energy (a few $10^{53} \mathrm{erg}$ ), and it could be the energy source of some of the long gamma ray bursts.

Aims. The main goal of the present work is to establish whether a newborn hadronic star (proto-hadronic star) could survive the early stages of its evolution without "decaying" to a quark star. To this aim, we study the nucleation process of quark matter in hot $(T \neq 0)$ $\beta$-stable hadronic matter, with and without trapped neutrinos, using a finite temperature equation of state (EOS) for hadronic and quark matter.

Methods. The finite-temperature EOS for the hadronic and for the quark phases were calculated using the nonlinear Walecka model and the MIT bag model, respectively. The quantum nucleation rate was calculated making use of the Lifshitz \& Kagan nucleation theory. The thermal nucleation rate was calculated using the Langer nucleation theory.

Results. We calculate and compare the nucleation rate and the nucleation time due to thermal and quantum nucleation mechanisms. We compute the crossover temperature above which thermal nucleation dominates the finite temperature quantum nucleation mechanism. We next discuss the consequences of quark matter nucleation for the physics and the evolution of proto-neutron stars. We introduce the new concept of limiting conversion temperature and critical mass $M_{\mathrm{cr}}$ for proto-hadronic stars, and we show that protohadronic stars with a mass $M<M_{\mathrm{cr}}$ could survive the early stages of their evolution without decaying to a quark star. We extend the concept of maximum mass of a "neutron star" with respect to the classical one introduced by Oppenheimer \& Volkoff to account for the existence of two distinct families of compact stars (hadronic stars and quark stars) as predicted by the present scenario.
\end{abstract}

Key words. dense matter - equation of state - stars: neutron

\section{Introduction}

According to quantum chromodynamics (QCD) a phase transition from hadronic matter to a deconfined quark phase should occur at a density of a few times nuclear matter saturation density $\rho_{0} \sim 2.8 \times 10^{14} \mathrm{~g} / \mathrm{cm}^{3}$. Neutron star structure calculations, based on a wide variety of modern equations of state (EOS) of hadronic matter (Lattimer \& Prakash 2001; Bombaci 2007), predict a maximum stellar central density (the one for the maximum mass star configuration) in the range of 4-8 times $\rho_{0}$. Consequently, the core of neutron stars is one of the best candidates in the universe where a phase of strong interacting matter with deconfined up, down and strange quarks could be found.

In the region of high density (high baryon chemical potential) and low temperature (which is the one relevant for neutron star physics) many QCD-inspired models suggest the deconfinement transition to be a first-order phase transition (Hsu \& Schwetz 1998; Fodor \& Katz 2004). As is well known, first-order phase transitions are triggered by the nucleation of a critical size drop of the new (stable) phase in a metastable mother phase.

In recent years there has been a growing interest in studying the nucleation process of quark matter in the core of massive neutron stars. The nucleation rate of quark matter in cold $(T=0) \beta$-stable nuclear matter has been calculated by Iida \& Sato (1997) wthin the Lifshitz-Kagan quantum nucleation theory (Lifshitz \& Kagan 1972). The same authors (Iida \& Sato 1998) have later on studied quark matter nucleation in $\beta$-stable hyperonic matter within a relativistic extension of the Lifshitz-Kagan theory. Various significant astrophysical implications of quark matter nucleation in neutron stars have been explored in a series of subsequent papers (Berezhiani et al. 2002, 2003; Bombaci et al. 2004; Drago et al. 2004; Lugones \& Bombaci 2005; Bombaci et al. 2007, 2008; Drago et al. 2008; Bambi \& Drago 2008). In particular, in these studies it has been shown that, above a threshold value of the central pressure, a pure hadronic compact star (HS) is metastable to the decay (conversion) to a quark star (QS), i.e. to a hybrid neutron star or to a strange star 
(Bodmer 1971; Witten 1984), depending on the details of the EOS for quark matter used to model the phase transition. This stellar conversion process releases (Bombaci \& Datta 2000) an extraordinarily large amount of energy (a few $10^{53} \mathrm{erg}$ ) and it could be the energy source of some of the long gamma ray bursts (GRBs).

The research reported in these papers has focused on the quark deconfinement phase transition in cold and neutrino-free neutron stars. In this case the formation of the first drop of QM could take place solely via a quantum nucleation process.

A neutron star at birth (proto-neutron star) is very hot $(T=$ 10-30 MeV) with neutrinos being still trapped in the stellar interior (Burrow \& Lattimer 1986; Prakash et al. 1997; Pons et al. 1999). Subsequent neutrino diffusion causes deleptonization and heats the stellar matter to an approximately uniform entropy per baryon $\tilde{S}=1-2$ (in units of the Boltzmann's constant $k_{\mathrm{B}}$ ). Depending on the stellar composition, during this stage neutrino escape can lead the more "massive" stellar configurations to the formation of a black hole (Bombaci 1996; Prakash et al. 1997). However, if the mass of the star is sufficiently low, the star will remain stable and will cool to temperatures well below $1 \mathrm{MeV}$ within a cooling time $t_{\text {cool }} \sim$ a few $10^{2} \mathrm{~s}$, as the neutrinos continue to carry energy away from the stellar material (Burrow \& Lattimer 1986; Prakash et al. 1997; Pons et al. 1999). Thus in a proto-neutron star, the quark deconfinement phase transition will likely be triggered by a thermal nucleation process. In fact, for sufficiently high temperatures, thermal nucleation is a much more efficient process than the quantum nucleation mechanism.

Some of the earlier studies of quark matter nucleation (see e.g., Horvath et al. 1992; Horvath 1994; Olesen \& Madsen 1994; Heiselberg 1995; Harko et al. 2004) have already dealt with thermal nucleation in hot and dense hadronic matter. In these studies, it has been found that the prompt formation of a critical size drop of quark matter via thermal activation is possible above a temperature of about 2-3 MeV. As a consequence, it was inferred that pure hadronic stars are converted to quark stars within the first seconds after their birth. However, these works (Horvath et al. 1992; Horvath 1994; Olesen \& Madsen 1994; Heiselberg 1995) reported an estimate of the thermal nucleation based on "typical" values for the thermodynamic properties characterizing the central part of neutron stars. Quark matter nucleation during the early post-bounce stage of core collapse supernova has been lately examined by Sagert et al. (2009) and Mintz et al. (2010).

The main goal of the present paper is to establish whether a newborn hadronic star (proto-hadronic star) could survive the early stages of its evolution without "decaying" to a quark star. To this aim, we calculated the thermal nucleation rate of quark matter in hot $(T \neq 0) \beta$-stable hadronic matter using a finite temperature EOS for hadronic and quark matter. In addition, we calculated the quantum nucleation rate at finite temperature, and compared the thermal and quantum nucleation time at different temperatures and pressures characterizing the central conditions of metastable proto-hadronic compact stars. We computed the crossover temperature above which thermal nucleation dominates the finite temperature quantum nucleation mechanism. An exploratory study of this issue has been recently reported (Bombaci et al. 2009) for the case of neutrino-free hadronic matter. Here, we extend our analysis and our numerical investigation to the case of hadronic matter with trapped neutrinos, and we make a systematic study of the finite temperature nucleation process on the value of the Bag constant in the EOS for the quark phase. Finally, we discuss the relevant consequences of quark matter nucleation for the evolution of proto-neutron stars.

\section{Equation of state}

Within a fundamental approach, the EOS for neutron star matter should be derived by solving numerically the equations of QCD on a space-time lattice. Lattice calculations at zero baryon chemical potential (zero baryon density) suggest that at high temperature and for physical values of the quark masses, the transition to quark gluon plasma is a "crossover" (Fodor \& Katz 2004; Karsch 2005) rather than a phase transition, which would be signaled by singularities in the thermodynamic observables. Unfortunately, present lattice QCD calculations at finite density (baryon chemical potential) are plagued with the notorius "sign problem", which makes them unrealizable by all presently known lattice methods (see e.g. Lombardo 2007, and references therein). Thus, to explore the QCD phase diagram at low temperature T and high baryon chemical potential $\mu$, it is necessary to invoke some approximations in QCD or to apply some QCD effective model. In this region of the $T-\mu$ plane, many QCD inspired models suggest the deconfinement transition to be a first-order phase transition (Hsu \& Schwetz 1998; Fodor \& Katz 2004). In this domain of the QCD phase diagram, many possible color superconducting phases of quark matter are expected (see e.g. Casalbuoni \& Nardulli 2004; Alford et al. 2008, and references therein). The effects of these color superconducting phases on the properties of proto-neutron stars have been investigated in (Aguilera et al. 2004; Sandin \& Blaschke 2007; Gu et al. 2008; Lugones et al. 2009).

Very recently, a new phase of QCD, named quarkyonic phase, has been predicted (McLerran \& Pisarski 2007; Hidaka et al. 2008). This matter phase is characterized by chiral symmetry and confinement.

Here, we have adopted a more traditional view, assuming a single first-order phase transition between the confined (hadronic) and deconfined phase of dense matter, and we used rather common models for describing them.

For the hadronic phase we used the nonlinear Walecka model (NLWM) (Walecka 1974; Serot \& Walecka 1986). This model is based on a relativistic Lagrangian of baryons interacting via the exchange of various mesons. We considered a version of the NLWM in which the baryon octet particles $\left(n, p, \Lambda, \Sigma^{-}, \Sigma^{0}, \Sigma^{+}\right.$, $\left.\Xi^{-}, \Xi^{0}\right)$ interact via the exchange of the scalar $\sigma$, the vectorisoscalar $\omega_{\mu}$ and the vector-isovector $\boldsymbol{\rho}_{\mu}$ meson fields (see e.g. Prakash et al. 1997; Glendenning 2000; Menezes \& Providência 2003).

The Lagrangian density of the model reads as

$\mathcal{L}=\mathcal{L}_{\text {hadrons }}+\mathcal{L}_{\text {leptons }}$

where the hadronic contribution is

$\mathcal{L}_{\text {hadrons }}=\mathcal{L}_{\text {baryons }}+\mathcal{L}_{\text {mesons }}$

with

$\mathcal{L}_{\text {baryons }}=\sum_{\text {baryons }} \bar{\psi}\left[\gamma^{\mu} D_{\mu}-M_{B}^{*}\right] \psi$,

where

$D_{\mu}=\mathrm{i} \partial_{\mu}-g_{\omega B} \omega_{\mu}-g_{\rho B} \boldsymbol{t}_{B} \cdot \boldsymbol{\rho}_{\mu}$,

and $M_{B}^{*}=M_{B}-g_{\sigma B} \sigma$. The quantity $\boldsymbol{t}_{B}$ designates the isospin of baryon $B$. The mesonic contribution reads as

$\mathcal{L}_{\text {mesons }}=\mathcal{L}_{\sigma}+\mathcal{L}_{\omega}+\mathcal{L}_{\rho}$, 
with

$\mathcal{L}_{\sigma}=\frac{1}{2}\left(\partial_{\mu} \sigma \partial^{\mu} \sigma-m_{\sigma}^{2} \sigma^{2}\right)+\frac{1}{3 !} \kappa \sigma^{3}+\frac{1}{4 !} \lambda \sigma^{4}$

$\mathcal{L}_{\omega}=-\frac{1}{4} \Omega_{\mu \nu} \Omega^{\mu v}+\frac{1}{2} m_{\omega}^{2} \omega_{\mu} \omega^{\mu}, \quad \Omega_{\mu \nu}=\partial_{\mu} \omega_{v}-\partial_{\nu} \omega_{\mu}$,

$\mathcal{L}_{\rho}=-\frac{1}{4} \boldsymbol{B}_{\mu \nu} \cdot \boldsymbol{B}^{\mu v}+\frac{1}{2} m_{\rho}^{2} \boldsymbol{\rho}_{\mu} \cdot \boldsymbol{\rho}^{\mu}, \quad \boldsymbol{B}_{\mu v}=\partial_{\mu} \boldsymbol{\rho}_{v}-\partial_{\nu} \boldsymbol{\rho}_{\mu}-g_{\rho}\left(\boldsymbol{\rho}_{\mu} \times \boldsymbol{\rho}_{v}\right)$

for the lepton contribution we take

$\mathcal{L}_{\text {leptons }}=\sum_{\text {leptons }} \bar{\psi}_{l}\left(\mathrm{i} \gamma_{\mu} \partial^{\mu}-m_{l}\right) \psi_{l}$

where the sum is over electrons, muons and neutrinos for matter with trapped neutrinos. In uniform matter, we get for the baryon Fermi energy $\epsilon_{\mathrm{F} B}=g_{\omega B} \omega_{0}+g_{\rho B} t_{3 B} \rho_{03}+\sqrt{k_{\mathrm{F} B}^{2}+M_{B}^{* 2}}$, with the baryon effective mass $M_{B}^{*}=M-g_{\sigma B} \sigma$.

In the present work we used one of the parametrizations of the NLWM given by Glendenning \& Moszkowski (2001) (hereafter the GM1 equation of state). The parameters of the GM1 EOS are fitted to the saturation properties of symmetric nuclear matter: binding energy per nucleon $B / A=-16.3 \mathrm{MeV}$, saturation density $\rho_{0}=0.153 \mathrm{fm}^{-3}$, incompressibility $K=300 \mathrm{MeV}$, nucleon effective mass $M^{*}=0.7$, and to the nuclear symmetry energy at saturation density $a_{\text {sym }}=32.5 \mathrm{MeV}$.

Including hyperons involves new couplings, i.e., the hyperon-nucleon couplings: $g_{\sigma B}=x_{\sigma B} g_{\sigma}, \quad g_{\omega B}=$ $x_{\omega B} g_{\omega}, g_{\rho B}=x_{\rho B} g_{\rho}$. For nucleons we take $x_{\sigma B}, x_{\omega B}, x_{\rho B}=1$ and for hyperons we will consider the couplings proposed by Glendenning \& Moszkowski (2001). They consider the binding energy of the $\Lambda$ in nuclear matter, $B_{\Lambda}$,

$\left(\frac{B_{\Lambda}}{A}\right)=-28 \mathrm{MeV}=x_{\omega} g_{\omega} \omega_{0}-x_{\sigma} g_{\sigma} \sigma$

to establish a relation between $x_{\sigma}$ and $x_{\omega}$. Moreover, known neutron star masses restrict $x_{\sigma}$ to the range 0.6-0.8. We take $x_{\rho}=x_{\sigma}$ and consider $x_{\sigma}=0.6$.

For the quark phase, we adopted a phenomenological EOS (Farhi \& Jaffe 1984) that is based on the MIT bag model for hadrons and considered different possible values for the bag constant B. The remaining model parameters have been fixed to: $m_{\mathrm{u}}=m_{\mathrm{d}}=0, m_{\mathrm{s}}=150 \mathrm{MeV}$ for the masses of the up, down, and strange quark, respectively, and $\alpha_{\mathrm{s}}=0$ for the QCD structure constant.

The two models for the EOS have been generalized to the case of finite temperature (see e.g. Menezes \& Providência 2003).

\section{Phase equilibrium}

For a first-order phase transition, the conditions for phase equilibrium are given by the Gibbs' phase rule

$T_{\mathrm{H}}=T_{\mathrm{Q}} \equiv T, \quad P_{\mathrm{H}}=P_{\mathrm{Q}} \equiv P_{0}$,

$\mu_{\mathrm{H}}\left(T, P_{0}\right)=\mu_{\mathrm{Q}}\left(T, P_{0}\right)$,

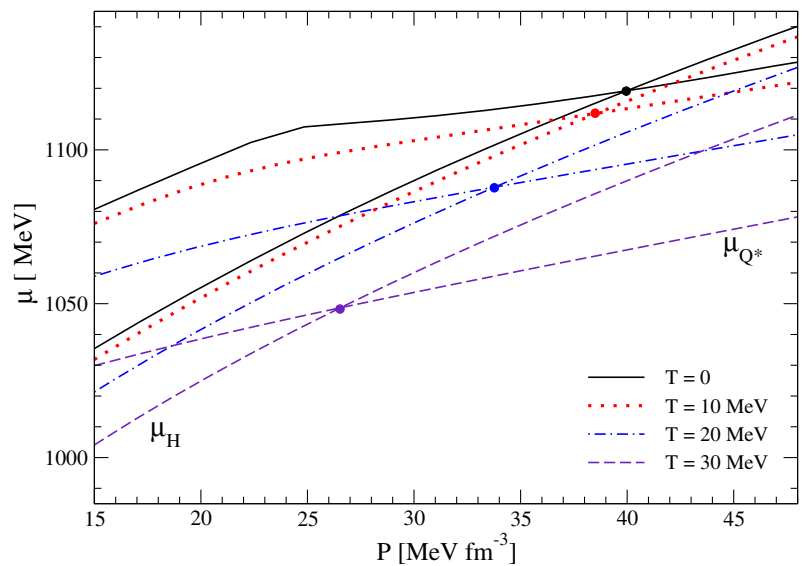

Fig. 1. (Color online) Gibbs energy per baryon, in neutrino-free matter, as a function of pressure for the hadronic and quark phases at different temperatures. Lines with the steeper slope refer to the hadronic phase. Full dots indicate the transition pressure $P_{0}$ for each temperature. GM1 EOS for the hadronic phase and MIT bag model EOS with $B=85 \mathrm{MeV} / \mathrm{fm}^{3}$ for the quark phase.

where

$\mu_{\mathrm{H}}=\frac{\varepsilon_{\mathrm{H}}+P_{\mathrm{H}}-s_{\mathrm{H}} T}{n_{\mathrm{H}}}, \mu_{\mathrm{Q}}=\frac{\varepsilon_{\mathrm{Q}}+P_{\mathrm{Q}}-s_{\mathrm{Q}} T}{n_{\mathrm{Q}}}$

are the Gibbs' energies per baryon (average chemical potentials) for the hadron and quark phase respectively, and $\varepsilon_{\mathrm{H}}\left(\varepsilon_{\mathrm{Q}}\right)$, $P_{\mathrm{H}}\left(P_{\mathrm{Q}}\right), s_{\mathrm{H}}\left(s_{\mathrm{Q}}\right)$ and $n_{\mathrm{H}}\left(n_{\mathrm{Q}}\right)$ respectively denote the total (i.e., including leptonic contributions) energy density, total pressure, total entropy density, and baryon number density for the hadron (quark) phase. Above the "transition point" $\left(P_{0}\right)$, the hadronic phase is metastable, and the stable quark phase will appear as a result of a nucleation process.

Small localized fluctuations in the state variables of the metastable hadronic phase will give rise to virtual drops of the stable quark phase. These fluctuations are characterized by a time scale $v_{0}^{-1} \sim 10^{-23} \mathrm{~s}$. It is set by the strong interactions (responsible for the deconfinement phase transition), and it is many orders of magnitude shorter than the typical time scale for the weak interactions. Quark flavor must therefore be conserved during the deconfinement transition. We refer to this form of deconfined matter, in which the flavor content is equal to that of the $\beta$-stable hadronic system at the same pressure and temperature, as the $Q^{*}$-phase. Soon after a critical size drop of quark matter is formed, the weak interactions will have enough time to act, changing the quark flavor fraction of the deconfined droplet to lower its energy, and a droplet of $\beta$-stable quark matter is formed (hereafter the Q-phase).

This first seed of quark matter will trigger the conversion (Olinto 1987; Heiselberg et al. 1991; Bombaci \& Datta 2000; Drago et al. 2010) of the pure hadronic star to a hybrid star or to a strange star. Thus, pure hadronic stars with values of the central pressure higher than $P_{0}$ are metastable to the decay (conversion) to hybrid stars or to strange stars (Berezhiani et al. 2002, 2003; Bombaci et al. 2004; Drago et al. 2004; Lugones \& Bombaci 2005; Bombaci et al. 2007). The mean lifetime of the metastable stellar configuration is related to the time needed to nucleate the first drop of quark matter in the stellar center, and it depends dramatically on the value of the stellar central pressure.

In Figs. 1 and 2, we plot the Gibbs' energies per baryon for the hadron-phase and the $\mathrm{Q}^{*}$-phase in neutrino-free matter, at different temperatures $(T=0,10,20,30 \mathrm{MeV})$ and for two different values of the bag constant $B=85 \mathrm{MeV} / \mathrm{fm}^{3}$ (Fig. 1) and 


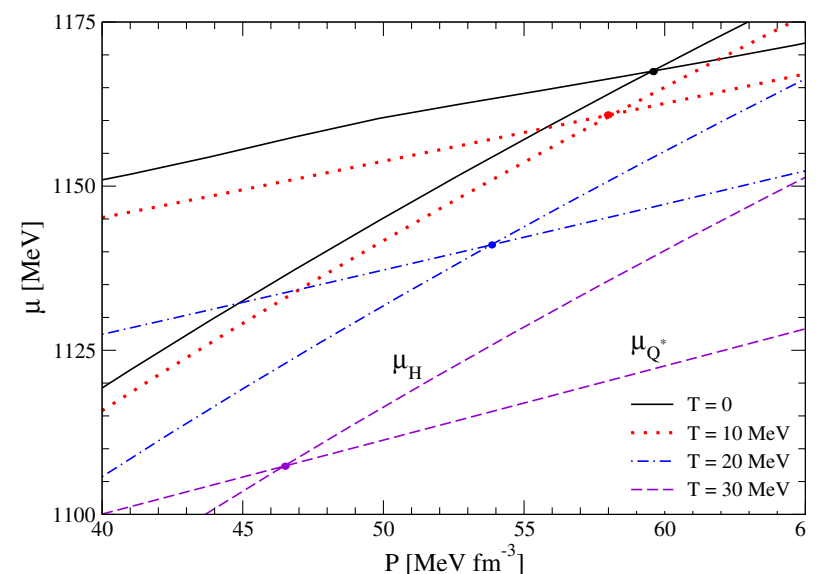

Fig. 2. Same as Fig. 1, but with a bag constant $B=100 \mathrm{MeV} / \mathrm{fm}^{3}$. (Color online)

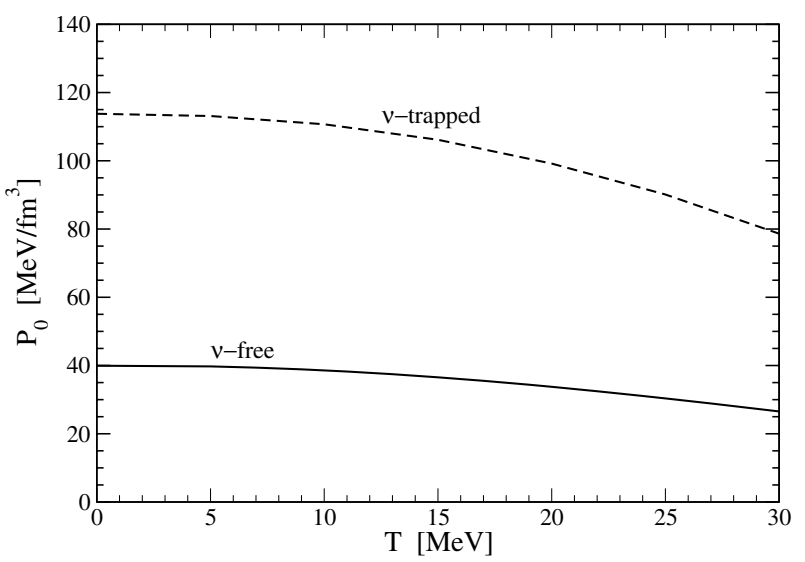

Fig. 3. Phase equilibrium curve for the hadron to $Q^{*}$-matter phase transition. The continuous curve is relative to neutrino-free matter, the dashed curve to matter with trapped neutrinos. EOS: GM1 plus MIT Bag model with $B=85 \mathrm{MeV} / \mathrm{fm}^{3}$.

$B=100 \mathrm{MeV} / \mathrm{fm}^{3}$ (Fig. 2). Lines with the steeper slope refer to the hadron phase. As we see, the transition pressure $P_{0}$ (indicated by a full dot) decreases when the hadronic matter temperature is increased.

The phase equilibrium curve $P_{0}(T)$ for the hadron-quark phase transition (in the case $B=85 \mathrm{MeV} / \mathrm{fm}^{3}$ ) is shown in Fig. 3 for neutrino-free matter and matter with trapped neutrinos. The region of the $P_{0}-T$ plane above each curve represents the deconfined Q*-phase. As expected (Prakash et al. 1997; Lugones \& Benvenuto 1998; Vidaña et al. 2005; Lugones et al. 2009) neutrino trapping in $\beta$-stable hadronic matter inhibits the quark deconfinement phase transition, thus the global effect of neutrino-trapping is to produce a shift of the phase equilibrium curve toward higher values of the pressure in the $P_{0}-T$ plane.

As is well known, for a first-order phase transition the derivative $\mathrm{d} P_{0} / \mathrm{d} T$ is related to the specific (i.e. per baryon) latent heat $Q$ of the phase transition by the Clapeyron-Clausius equation

$\frac{\mathrm{d} P_{0}}{\mathrm{~d} T}=-\frac{n_{\mathrm{H}} n_{\mathrm{Q}^{*}}}{n_{\mathrm{Q}^{*}}-n_{\mathrm{H}}} \frac{Q}{T}$

$Q=\tilde{W}_{\mathrm{Q}^{*}}-\tilde{W}_{\mathrm{H}}=T\left(\tilde{S}_{\mathrm{Q}^{*}}-\tilde{S}_{\mathrm{H}}\right)$

where $\tilde{W}_{\mathrm{H}}\left(\tilde{W}_{\mathrm{Q}^{*}}\right)$ and $\tilde{S}_{\mathrm{H}}\left(\tilde{S}_{\mathrm{Q}^{*}}\right)$ denote the enthalpy per baryon and entropy per baryon for the hadron (quark) phase,
Table 1. The specific latent heat $Q$ and the phase number densities $n_{\mathrm{H}}$ and $n_{\mathrm{Q}^{*}}$ at phase equilibrium.

\begin{tabular}{lcccc}
\hline \hline$T$ \\
$\mathrm{MeV}$ & $\begin{array}{c}\mathrm{Q} \\
\mathrm{MeV}\end{array}$ & $\begin{array}{c}n_{\mathrm{Q}^{*}} \\
\mathrm{fm}^{-3}\end{array}$ & $\begin{array}{c}n_{\mathrm{H}} \\
\mathrm{fm}^{-3}\end{array}$ & $\begin{array}{c}P_{0} \\
\mathrm{MeV} / \mathrm{fm}^{3}\end{array}$ \\
\hline 0 & 0.00 & 0.453 & 0.366 & 39.95 \\
5 & 0.56 & 0.451 & 0.364 & 39.74 \\
10 & 2.40 & 0.447 & 0.358 & 38.58 \\
15 & 5.71 & 0.439 & 0.348 & 36.55 \\
20 & 10.60 & 0.428 & 0.334 & 33.77 \\
25 & 17.17 & 0.414 & 0.316 & 30.36 \\
30 & 25.44 & 0.398 & 0.294 & 26.53 \\
\hline
\end{tabular}

Notes. GM1 EOS for the hadronic phase, MIT bag model for the quark phase with $B=85 \mathrm{MeV} / \mathrm{fm}^{3}$. Results for neutrino-free matter.

Table 2. Same as Table 1 , but with $B=100 \mathrm{MeV} / \mathrm{fm}^{3}$.

\begin{tabular}{lcccc}
\hline \hline $\begin{array}{l}T \\
\mathrm{MeV}\end{array}$ & $\begin{array}{c}\mathrm{Q} \\
\mathrm{MeV}\end{array}$ & $\begin{array}{c}n_{\mathrm{Q}^{*}} \\
\mathrm{fm}^{-3}\end{array}$ & $\begin{array}{c}n_{\mathrm{H}} \\
\mathrm{fm}^{-3}\end{array}$ & $\begin{array}{c}P_{0} \\
\mathrm{MeV} / \mathrm{fm}^{3}\end{array}$ \\
\hline 0 & 0.00 & 0.557 & 0.447 & 59.53 \\
5 & 0.44 & 0.556 & 0.445 & 59.22 \\
10 & 1.98 & 0.552 & 0.441 & 58.18 \\
15 & 4.91 & 0.544 & 0.432 & 56.39 \\
20 & 9.35 & 0.533 & 0.421 & 53.81 \\
25 & 15.49 & 0.519 & 0.401 & 50.47 \\
30 & 23.51 & 0.503 & 0.386 & 46.44 \\
\hline
\end{tabular}

Table 3. Same as Table 1, but with trapped neutrinos.

\begin{tabular}{lcccc}
\hline \hline$T$ & $\begin{array}{c}Q \\
\mathrm{MeV}\end{array}$ & $\begin{array}{c}n_{\mathrm{Q}^{*}} \\
\mathrm{fm}^{-3}\end{array}$ & $\begin{array}{c}n_{\mathrm{H}} \\
\mathrm{fm}^{-3}\end{array}$ & $\begin{array}{c}P_{0} \\
\mathrm{MeV} / \mathrm{fm}^{3}\end{array}$ \\
\hline 0 & 0.00 & 0.603 & 0.516 & 113.77 \\
5 & 0.65 & 0.601 & 0.514 & 113.11 \\
10 & 2.87 & 0.594 & 0.509 & 110.69 \\
15 & 6.78 & 0.580 & 0.499 & 106.17 \\
20 & 12.65 & 0.560 & 0.483 & 99.18 \\
25 & 20.21 & 0.534 & 0.462 & 90.12 \\
30 & 29.88 & 0.502 & 0.434 & 78.65 \\
\hline
\end{tabular}

respectively. The specific latent heat $Q$ and the phase numbers densities $n_{\mathrm{H}}$ and $n_{\mathrm{Q}^{*}}$ at phase equilibrium are reported in Tables 1 and 2 for the case of neutrino-free matter and for two different values of the bag constant $B=85 \mathrm{MeV} / \mathrm{fm}^{3}$ (Table 1) and $B=100 \mathrm{MeV} / \mathrm{fm}^{3}$ (Table 2). As expected for a first-order phase transition, one has a discontinuity jump in the phase number densities: in our particular case $n_{\mathrm{Q}^{*}}\left(T, P_{0}\right)>n_{\mathrm{H}}\left(T, P_{0}\right)$. This result, together with the positive value of $Q$ (i.e. the deconfinement phase transition absorbs heat), tells us (see Eq. (14)) that the phase transition temperature (11) decreases with pressure (as in the melting of ice).

To explore the effect of neutrino trapping on the phase equilibrium properties of the system, in Table 3 we report the quantities $Q, n_{\mathrm{H}}, n_{\mathrm{Q}^{*}}$ and $P_{0}$ at different temperatures, for a bag constant $B=85 \mathrm{MeV} / \mathrm{fm}^{3}$ (compare with the results in Table 1). As we see, the phase number densities $n_{\mathrm{H}}$ and $n_{\mathrm{Q}^{*}}$ at phase equilibrium are shifted to higher values, and the specific latent heat $Q$ is increased with respect to the neutrino-free matter case.

We also made systematic calculations of the phase equilibrium properties varying the value of the bag constant in the EOS of the $Q^{*}$-phase. These results are summarized in Fig. 4 where we plot the phase equilibrium pressure $P_{0}$ between the hadron phase and the $Q^{*}$ phase at zero temperature as a function of $B$ for neutrino-free matter and neutrino-trapped matter. As expected, a 
I. Bombaci et al.: Effects of quark matter nucleation on the evolution of proto-neutron stars

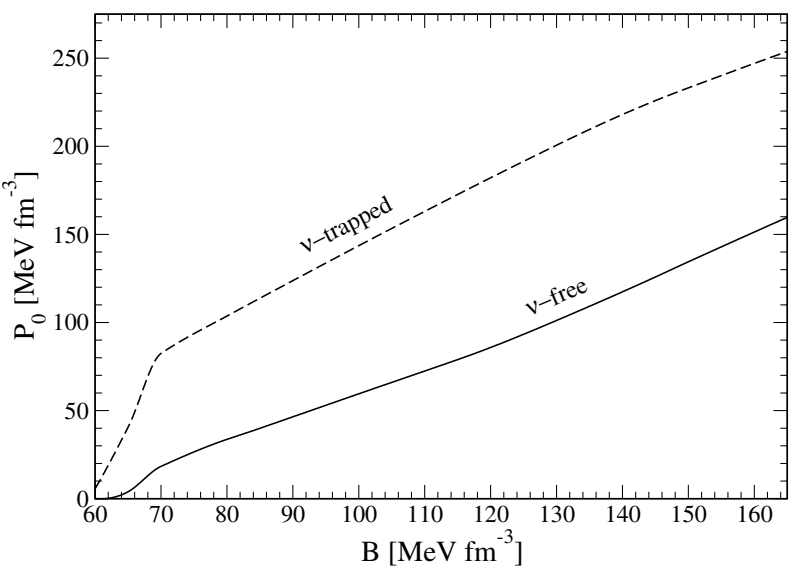

Fig. 4. Phase equilibrium pressure $P_{0}$ between the hadron phase and the $\mathrm{Q}^{*}$ phase at $T=0$, as a function of the bag constant $B$. The continuous curve refers to neutrino-free matter, and the dashed curve refers to neutrino-trapped matter.

higher value of $B$ makes the deconfinemnt transition more difficult, increasing the value of the transition pressure.

\section{Quantum and thermal nucleation rates}

Above the phase equilibrium pressure $P_{0}$ the $\beta$-stable hadron phase is metastable, and the formation of the stable (with respect to the strong interactions) $Q^{*}$-phase will occur via a nucleation process. The main effect of finite temperature on the quantum nucleation mechanism of quark matter is to modify the energy barrier separating the quark phase from the metastable hadronic phase. This energy barrier, which represents the difference in the free energy of the system with and without a $Q^{*}$-matter droplet, can be written as

$$
U(\mathcal{R}, T)=\frac{4}{3} \pi n_{\mathrm{Q}^{*}}\left(\mu_{\mathrm{Q}^{*}}-\mu_{\mathrm{H}}\right) \mathcal{R}^{3}+4 \pi \sigma \mathcal{R}^{2}
$$

where $\mathcal{R}$ is the radius of the droplet (supposed to be spherical), and $\sigma$ is the surface tension for the surface separating the hadron from the $\mathrm{Q}^{*}$-phase. The energy barrier has a maximum at the critical radius $\mathcal{R}_{\mathrm{c}}=2 \sigma /\left[n_{\mathrm{Q}^{*}}\left(\mu_{\mathrm{H}}-\mu_{\mathrm{Q}^{*}}\right)\right]$. We neglected the term associated with the curvature energy, and also the terms connected with the electrostatic energy, since they are known to only introduce small corrections (Iida \& Sato 1998; Bombaci et al. 2004). The value of the surface tension $\sigma$ for the interface separating the quark and hadron phase is poorly known, and typically values used in the literature range within $10-50 \mathrm{MeV} \mathrm{fm}^{-2}$ (Heiselberg et al. 1993; Iida \& Sato 1998). We assume $\sigma$ to be temperature independent and we take $\sigma=30 \mathrm{MeV} \mathrm{fm}^{-2}$.

The quantum nucleation time $\tau_{\mathrm{q}}$ can be straightforwardly evaluated within a semi-classical approach (Lifshitz \& Kagan 1972; Iida \& Sato 1997, 1998). First one computes the ground state energy $E_{0}$ in the WKB approximation and the oscillation frequency $v_{0}$ of the drop in the potential well $U(\mathcal{R}, T)$. Then, the probability of tunneling is given by

$p_{0}=\exp \left[-\frac{A\left(E_{0}\right)}{\hbar}\right]$

where $A(E)$ is the action under the potential barrier, which in a relativistic framework reads as (Iida \& Sato 1998)

$$
A(E)=\frac{2}{c} \int_{\mathcal{R}_{-}}^{\mathcal{R}_{+}} \sqrt{\left[2 m(\mathcal{R}) c^{2}+E-U(\mathcal{R})\right][U(\mathcal{R})-E]}
$$

with $\mathcal{R}_{ \pm}$the classical turning points and $m(\mathcal{R})$ the droplet effective mass. The quantum nucleation time is then equal to

$\tau_{\mathrm{q}}=\left(v_{0} p_{0} N_{\mathrm{c}}\right)^{-1}$,

with $N_{\mathrm{c}} \sim 10^{48}$ the number of nucleation centers expected in the innermost part ( $r \leq R_{\text {nuc }} \sim 100 \mathrm{~m}$ ) of the hadronic star, where the pressure and temperature can be considered constant and equal to their central values.

The thermal nucleation rate can be written (Langer \& Turski 1973) as

$I=\frac{\kappa}{2 \pi} \Omega_{0} \exp \left(-U\left(\mathcal{R}_{\mathrm{c}}, T\right) / T\right)$

where $\kappa$ is the so-called dynamical prefactor, which is related to the growth rate of the drop radius $\mathcal{R}$ near the critical radius $\left(\mathcal{R}_{\mathrm{c}}\right)$; $\Omega_{0}$ is the so-called statistical prefactor, which measures the phase-space volume of the saddle-point region around $\mathcal{R}_{\mathrm{c}}$; and $U\left(\mathcal{R}_{\mathrm{c}}, T\right)$ is the activation energy, i.e. the change in the free energy of the system required to activate the formation of a critical size droplet. The Langer theory (Langer 1968, 1969; Langer \& Turski 1973; Turski \& Langer 1980) of homogeneous nucleation has been extended in Csernai \& Kapusta (1992) and Venugopalan \& Vischer (1994) to the case of first-order phase transitions occurring in relativistic systems, as in the case of the quark deconfinement transition. The statistical prefactor can be written (Csernai \& Kapusta 1992) as

$\Omega_{0}=\frac{2}{3 \sqrt{3}}\left(\frac{\sigma}{T}\right)^{3 / 2}\left(\frac{\mathcal{R}}{\xi_{\mathrm{Q}}}\right)^{4}$

where $\xi_{\mathrm{Q}}$ is the quark correlation length, which gives a measure of the thickness of the interface layer between the two phases (the droplet "surface thickness"). In the present calculation, we take $\xi_{\mathrm{Q}}=0.7 \mathrm{fm}$ according to the estimate given in Csernai \& Kapusta (1992) and Heiselberg (1995). For the dynamical prefactor, we have used a general expression, which has been derived by Venugopalan \& Vischer (1994)

$\kappa=\frac{2 \sigma}{\mathcal{R}_{\mathrm{c}}^{3}(\Delta w)^{2}}\left[\lambda T+2\left(\frac{4}{3} \eta+\zeta\right)\right]$,

where $\Delta w=w_{\mathrm{Q} *}-w_{\mathrm{H}}$ is the difference between the enthalpy density of the two phases, $\lambda$ the thermal conductivity, and $\eta$ and $\zeta$ are the shear and bulk viscosities respectively of hadronic matter. The nucleation prefactor used in the present work differs significantly from the one used in previous works (Horvath et al. 1992; Horvath 1994; Olesen \& Madsen 1994), where based on dimensional grounds, the prefactor was taken to be equal to $T^{4}$.

There are not many calculations of the transport properties of dense hadronic matter. With a few exceptions (see e.g. van Dalen \& Dieperink 2004; Chattaerjee \& Bandyopadhyay 2006) most of them are relative to nuclear or pure neutron matter (Flowers \& Itho 1979; Danielewicz 1984; Sedrakian et al. 1994; Benhar \& Valli 2007; Shternin \& Yakovlev 2008; Benhar et al. 2010; Zhang et al. 2010). These quantities have been calculated by Danielewicz (1984) in the case of nuclear matter. According to the results of Danielewicz (1984), the dominant contribution to the prefactor $\kappa$ comes from the shear viscosity $\eta$. Therefore, we take $\lambda$ and $\zeta$ equal to zero, and we use the following relation for the shear viscosity (Danielewicz 1984):

$\eta=\frac{7.6 \times 10^{26}}{(T / \mathrm{MeV})^{2}}\left(\frac{n_{\mathrm{H}}}{n_{0}}\right)^{2} \frac{\mathrm{MeV}}{\mathrm{fm} \mathrm{s}}$, 


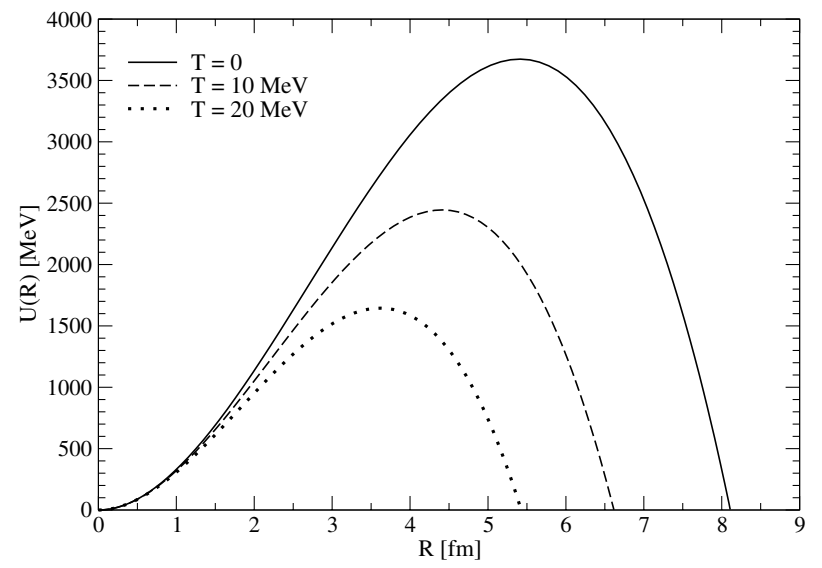

Fig. 5. Energy barrier for a virtual drop of the $\mathrm{Q}^{*}$-phase in $\beta$-stable neutrino-free hadronic matter as a function of the droplet radius and for different temperatures for a fixed pressure $P=57 \mathrm{MeV} / \mathrm{fm}^{3}$. The bag constant takes the value $B=85 \mathrm{MeV} / \mathrm{fm}^{3}$.

Table 4. Crossover temperature $T_{\text {co }}$ (in $\mathrm{MeV}$ ), for different fixed values of the pressure $P$ (in $\mathrm{MeV} / \mathrm{fm}^{3}$ ) of neutrino-free hadronic matter.

\begin{tabular}{lcc}
\hline \hline$P$ & $T_{\text {co }}$ & $\log _{10}(\tau / \mathrm{s})$ \\
\hline 53.98 & 5.0 & 233.6 \\
55.48 & 6.0 & 121.3 \\
56.94 & 7.0 & 56.6 \\
58.42 & 8.0 & 16.0 \\
58.85 & 8.3 & 3.0 \\
\hline
\end{tabular}

Notes. The third column reports the logarithm of the nucleation time (in seconds) calculated at the crossover temperature. The value $8.3 \mathrm{MeV}$ defines the value of the limiting conversion temperature $\Theta$ for a star with a central pressure $P=58.85 \mathrm{MeV} \mathrm{fm}^{-3}$.

with $n_{0}=0.16 \mathrm{fm}^{-3}$ the saturation density of normal nuclear matter.

The thermal nucleation time $\tau_{\text {th }}$, relative to the innermost stellar region $\left(V_{\text {nuc }}=(4 \pi / 3) R_{\text {nuc }}^{3}\right)$ where almost constant pressure and temperature occur, can thus be written as

$\tau_{\text {th }}=\left(V_{\text {nuc }} I\right)^{-1}$.

In Fig. 5, we represent the energy barrier for a virtual drop of the $\mathrm{Q}^{*}$-phase (with $B=85 \mathrm{MeV} / \mathrm{fm}^{3}$ ) in the neutrino-free hadronic phase as a function of the droplet radius and for different temperatures at a fixed pressure $P=57 \mathrm{MeV} / \mathrm{fm}^{3}$. As expected, from the results plotted in Fig. 1, the energy barrier $U(\mathcal{R}, T)$ and the droplet critical radius $\mathcal{R}_{\mathrm{c}}$ decrease as the matter temperature is increased. This effect favors the $\mathrm{Q}^{*}$-phase formation and, in particular, increases (decreases) the quantum nucleation rate (nucleation time $\tau_{\mathrm{q}}$ ) with respect to the corresponding quantities calculated at $T=0$.

In Fig. 6 we plot the quantum and thermal nucleation times of the $\mathrm{Q}^{*}$-phase (with $B=85 \mathrm{MeV} / \mathrm{fm}^{3}$ ) in $\beta$-stable neutrinofree hadronic matter as a function of temperature and at a fixed pressure $P=57 \mathrm{MeV} / \mathrm{fm}^{3}$. As expected, we find a crossover temperature $T_{\text {co }}$ above which thermal nucleation is dominant with respect to the quantum nucleation mechanism. For the case reported in Fig. 6, we have $T_{\mathrm{co}}=7.05 \mathrm{MeV}$ and the corresponding nucleation time is $\log _{10}(\tau / \mathrm{s})=54.4$. The crossover temperature for different values of the pressure of $\beta$-stable hadronic matter is reported in Table 4 (second column), together with the nucleation time calculated at $T=T_{\text {co }}$ (third column).

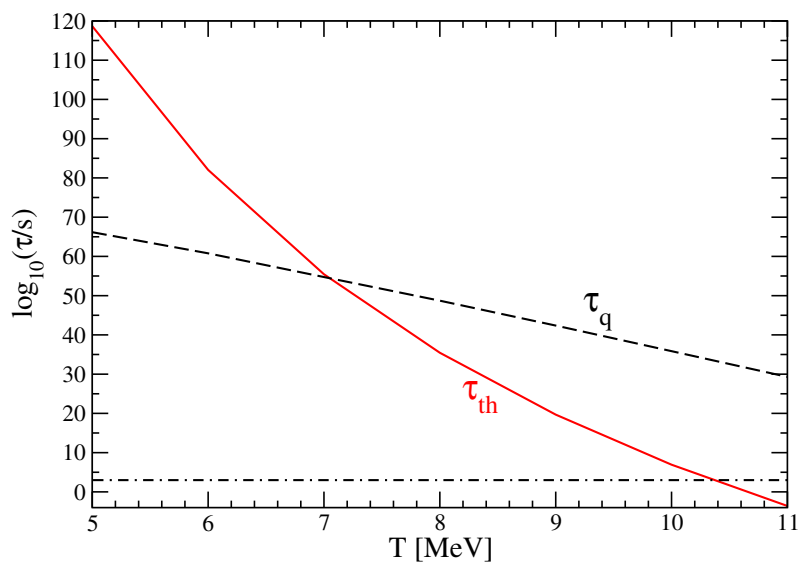

Fig. 6. (Color online) Thermal $\left(\tau_{\text {th }}\right)$ and quantum $\left(\tau_{\mathrm{q}}\right)$ nucleation time of the $\mathrm{Q}^{*}$-phase (with $B=85 \mathrm{MeV} / \mathrm{fm}^{3}$ ) in $\beta$-stable neutrino-free hadronic matter as a function of temperature at fixed pressure $P=57 \mathrm{MeV} / \mathrm{fm}^{3}$. The crossover temperature is $T_{\text {co }}=7.05 \mathrm{MeV}$. The limiting conversion temperature for the proto-hadronic star is, in this case, $\Theta=10.3 \mathrm{MeV}$, obtained from the intersection of the thermal nucleation time curve (continuous line) and the dot-dashed line representing $\log _{10}(\tau / \mathrm{s})=3$.

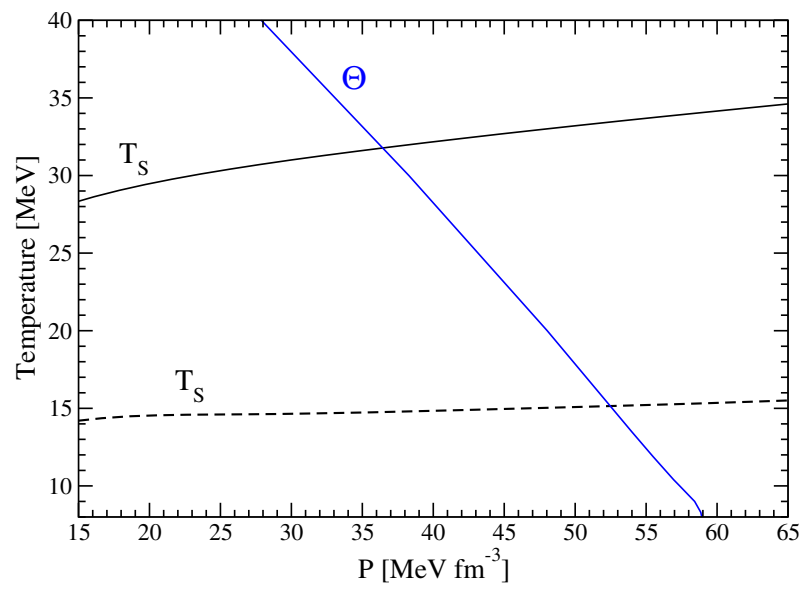

Fig. 7. (Color online) The limiting conversion temperature $\Theta$ for a newborn hadronic star as a function of the central stellar pressure. The lines labeled $T_{\mathrm{S}}$ represent the stellar matter temperature as a function of pressure at fixed entropies per baryon $\tilde{S} / k_{\mathrm{B}}=1$ (dashed line) and 2 (solid line). Results for neutrino-free matter.

\section{Evolution of proto-hadronic stars}

Keeping in mind the physical conditions in the interior of a proto-hadronic star (Burrow \& Lattimer 1986; Prakash et al. 1997) (see Sect. 1 of the present paper) to establish whether this star will survive the early stages of its evolution without "decaying" to a quark star, one has to compare the quark matter nucleation time $\tau=\min \left(\tau_{\mathrm{q}}, \tau_{\text {th }}\right)$ with the cooling time $t_{\text {cool }} \sim$ a few $10^{2}$ s. If $\tau \gg t_{\text {cool }}$ then quark matter nucleation is not likely to occur in the newly formed star, and this star will evolve into a cold deleptonized configuration. We thus introduce the concept of limiting conversion temperature $\Theta$ for the proto-hadronic star and define it as the value of the stellar central temperature $T_{\mathrm{c}}$ for which the $\mathrm{Q}^{*}$-matter nucleation time is equal to $10^{3} \mathrm{~s}$. The limiting conversion temperature $\Theta$ will clearly depend on the value of the stellar central pressure (and thus on the value of the stellar mass).

The limiting conversion temperature $\Theta$ is plotted in Fig. 7 as a function of the stellar central pressure $P$. A proto-hadronic star with a central temperature $T_{\mathrm{c}}>\Theta(P)$ will likely nucleate 
Table 5. Gravitational $\left(M_{\mathrm{cr}}\right)$ and baryonic $\left(M_{B, \mathrm{cr}}\right)$ critical mass (see text for more details) for proto-hadronic stars at different entropy per baryon $\tilde{S} / k_{\mathrm{B}}$.

\begin{tabular}{lccc}
\hline \hline$\tilde{S} / k_{\mathrm{B}}$ & $M_{\text {cr }}$ & $M_{B, \text { cr }}$ & $\mathcal{M}$ \\
\hline 0.0 & 1.573 & 1.752 & 1.573 \\
1.0 & 1.494 & 1.643 & 1.485 \\
2.0 & 1.390 & 1.492 & 1.361 \\
\hline
\end{tabular}

Notes. $\mathcal{M}$ denotes the gravitational mass of the cold hadronic configuration with the same stellar baryonic mass $\left(M_{B, \mathrm{cr}}\right)$. Stellar masses are in units of the solar mass, $M_{\text {sun }}=1.989 \times 10^{33} \mathrm{~g}$. Results are relative to a bag constant $B=85 \mathrm{MeV} / \mathrm{fm}^{3}$.

a $Q^{*}$-matter drop during the early stages of its evolution, will finally evolve to a cold and deleptonized quark star, or will collapse to a black hole (depending on the particular model adopted for the matter EOS).

For an isoentropic stellar core (Burrow \& Lattimer 1986; Prakash et al. 1997), the central temperature of the protohadronic star is given for the present EOS model by the lines labeled by $T_{\mathrm{S}}$ in Fig. 7 , relative to the case $\tilde{S}=1 k_{\mathrm{B}}$ and $\tilde{S}=2 k_{\mathrm{B}}$. The intersection point $\left(P_{\mathrm{S}}, \Theta_{\mathrm{S}}\right)$ between the two curves $\Theta(P)$ and $T_{\mathrm{S}}(P)$ thus gives the central pressure and temperature of the configuration that we denote as the critical mass configuration of the proto-hadronic stellar sequence. The value of the gravitational critical mass $M_{\mathrm{cr}}=M\left(P_{\mathrm{S}}, \Theta_{\mathrm{S}}\right)$ and baryonic critical mass $M_{B, \mathrm{cr}}$ are reported in Table 5 for three different choices of the entropy per baryon, $\tilde{S} / k_{\mathrm{B}}=0$ (corresponding to a cold hadronic star) ${ }^{1}$, 1 and 2 in the case of a bag constant $B=85 \mathrm{MeV} / \mathrm{fm}^{3}$ and for $\sigma=30 \mathrm{MeV} / \mathrm{fm}^{2}$. In the same table, we also report the value of the gravitational mass $\mathcal{M}$ of the cold hadronic star with baryonic mass equal to $M_{B, \mathrm{cr}}$. This configuration is stable $(\tau=\infty)$ with respect to $\mathrm{Q}^{*}$-matter nucleation in the case $\tilde{S} / k_{\mathrm{B}}=2$, and it is essentially stable (having a nucleation time enormously longer than the age of the universe) in the case $\tilde{S} / k_{\mathrm{B}}=1$.

The limiting conversion temperature $\Theta$ for a newborn hadronic star is plotted in Fig. 8 for different values of the bag constant. The increase in $\mathrm{B}$ produces a growth of the region of the $P-T$ plane where the proto-hadronic star could survive Q* nucleation and thus evolve to a cold hadronic star.

To explore the role of neutrino trapping on the limiting conversion temperature $\Theta$ and on the critical mass of the protohadronic star, we plot in Fig. 9 the results of our calculations for $\Theta$ and for the stellar matter temperature $T_{\mathrm{S}}$ at fixed entropies per baryon $\left(\tilde{S} / k_{\mathrm{B}}=1\right.$, and 2$)$ in the case of neutrino-free matter and neutrino-trapped matter. Results in Fig. 9 are relative to the GM1 EOS for the hadronic phase and the bag model EOS with $B=85 \mathrm{Mev} / \mathrm{fm}^{3}$ for the quark phase. As we see, neutrino trapping has a conspicuous effect on $\Theta$, and ultimately strongly

\footnotetext{
${ }^{1}$ In reference (Berezhiani et al. 2002, 2003; Bombaci et al. 2004; Drago et al. 2004; Lugones \& Bombaci 2005; Bombaci et al. 2007, 2008), the critical mass for cold $(T=0)$ metastable hadronic stars has been defined as the value of the gravitational mass for which the quantum nucleation time is equal to one year: $M_{\mathrm{cr}}(T=0)=M\left(\tau_{\mathrm{q}}=1 \mathrm{yr}\right)$. It is worth recalling that the nucleation time $\tau_{\mathrm{q}}$ is an extremely steep function of the hadronic star mass (Berezhiani et al. 2002, 2003; Bombaci et al. 2004), therefore the exact value of $\tau_{\mathrm{q}}$ chosen in the definition of $M_{\mathrm{cr}}(T=0)$ is not crucial (one must take a "reasonable low" value of $\tau_{\mathrm{q}}$, much shorter than the age of young pulsars as the Crab pulsar). We have verified that changing $\tau_{\mathrm{q}}$ from $1 \mathrm{yr}$ to $10^{3} \mathrm{~s}$ modifies $M_{\mathrm{cr}}(T=0)$ by $\sim 0.02 \%$. On the other hand, the nucleation time $\tau=\min \left(\tau_{\mathrm{q}}, \tau_{\text {th }}\right)$ entering in the definition of the critical mass of proto-hadronic stars $M_{\mathrm{cr}}(\tilde{S})$ must be comparable to the proto-hadronic star cooling time $t_{\text {cool }}$.
}

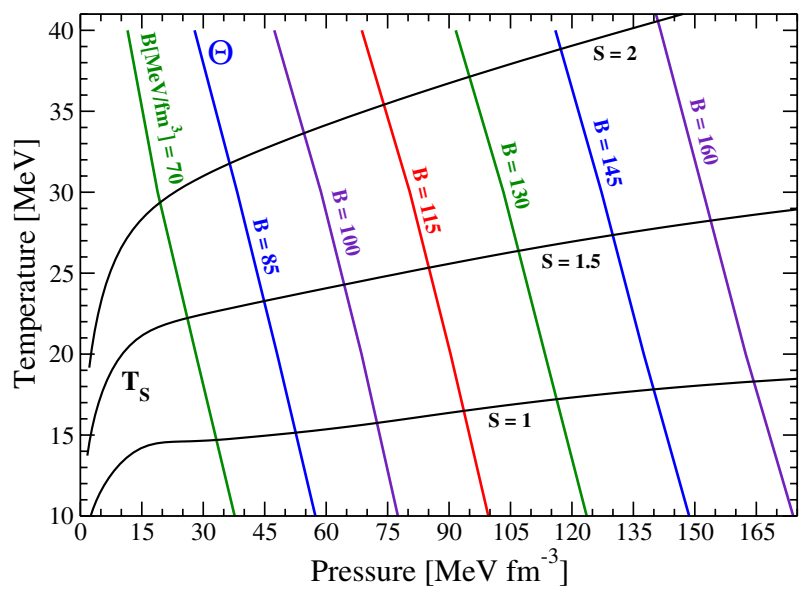

Fig. 8. (Color online) The limiting conversion temperature $\Theta$ for a newborn hadronic star as a function of the central stellar pressure for different values of the bag constant $B$. The lines labeled $T_{\mathrm{S}}$ represent the stellar matter temperature as a function of pressure at fixed entropies per baryon $\tilde{S} / k_{\mathrm{B}}=1,1.5,2$. Results for neutrino-free matter.

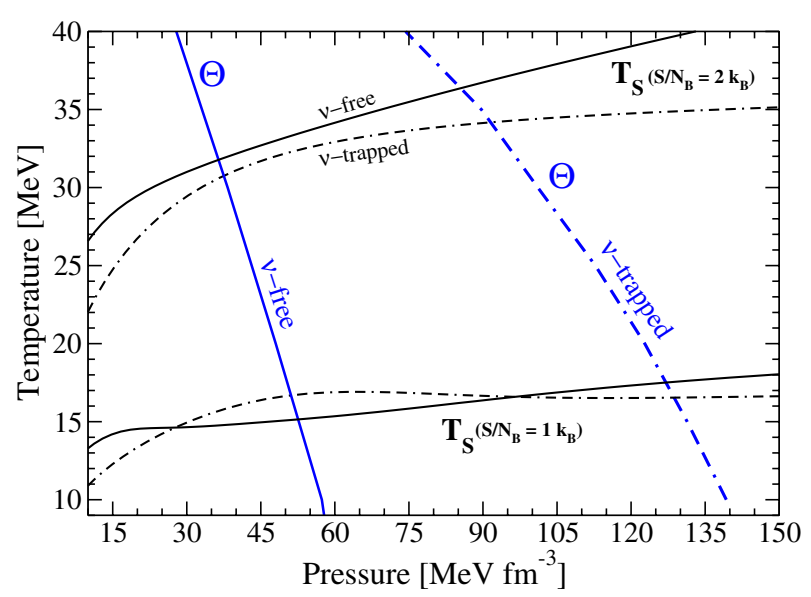

Fig. 9. (Color online) Effects of neutrino trapping on the limiting conversion temperature $\Theta$ for a newborn hadronic star as a function of the central stellar pressure.

suppresses the nucleation of $\mathrm{Q}^{*}$-matter in the proto-hadronic star. There is also a relatively small effect of neutrino trapping on $T_{\mathrm{S}}$.

The evolution of a proto-hadronic star (PHS) within our scenario is delineated in Fig. 10, where we plot the appropriate stellar equilibrium sequences in the gravitational-baryonic mass plane obained from the GM1 EOS for the hadronic phase and the bag model EOS with $B=85 \mathrm{MeV} / \mathrm{fm}^{3}$ for the quark phase. In particular, we plot the PHS sequence, i.e. isoentropic HSs $\left(\tilde{S}=2 k_{\mathrm{B}}\right)$ and neutrino-free matter (upper line), and the cold HS sequence (middle line). The asterisk and the full circle on these lines identify respectively the stellar configuration with $\tau=\infty$ and the critical mass configuration. We denote as $M_{B, \mathrm{cr}}^{\mathrm{PHS}} \equiv M_{B, \mathrm{cr}}\left(\tilde{S}=2 k_{\mathrm{B}}\right)$ the baryonic critical mass for the PHS sequence and as $M_{B, \mathrm{cr}}^{\mathrm{HS}} \equiv M_{B, \mathrm{cr}}(\tilde{S}=0)$ the baryonic critical mass for the cold hadronic star sequence. Finally, the lower line represents the cold QS sequence. We assume (Bombaci \& Datta 2000) $M_{B}=$ const. during these stages of the stellar evolution $)^{2}$. Thus

\footnotetext{
${ }^{2}$ Sizeable mass accretion on the proto-neutron star occurs within a time of $\sim 0.5 \mathrm{~s}$ after core bounce (Burrow \& Lattimer 1986; Prakash et al. 1997). During the subsequent stages, the star thus evolves with $M_{B} \simeq$ const.
} 


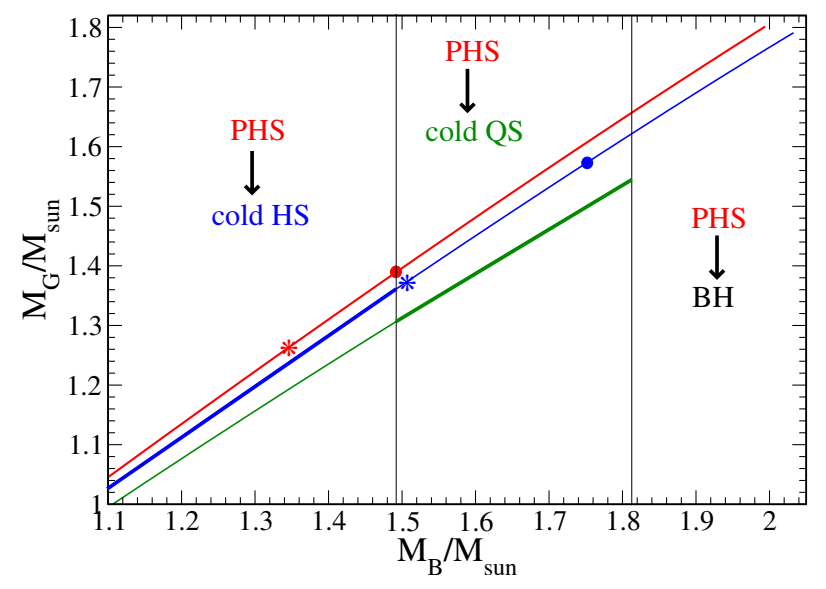

Fig. 10. (Color online) Evolution of a proto-hadronic star in the gravitational-baryonic mass plane for $B=85 \mathrm{MeV} / \mathrm{fm}^{3}$ and $\sigma=$ $30 \mathrm{MeV} / \mathrm{fm}^{2}$. The upper (red) line represents the stellar equilibrium sequence for neutrino-free proto-hadronic stars (PHS) with $\tilde{S}=2 k_{\mathrm{B}}$. The middle (blue) line represents the cold the HS sequence. The asterisk and the full circle on these lines represent the stellar configuration with nucleation time $\tau=\infty$ and the critical mass configuration $M_{\mathrm{cr}}$, respectively. The lower (green) line represent the cold QS sequence. Assuming $M_{B}=$ const., the evolution of a PHS in this plane occurs along a vertical line. Stellar masses are in units of the solar mass, $M_{\text {sun }}=1.989 \times 10^{33} \mathrm{~g}$.

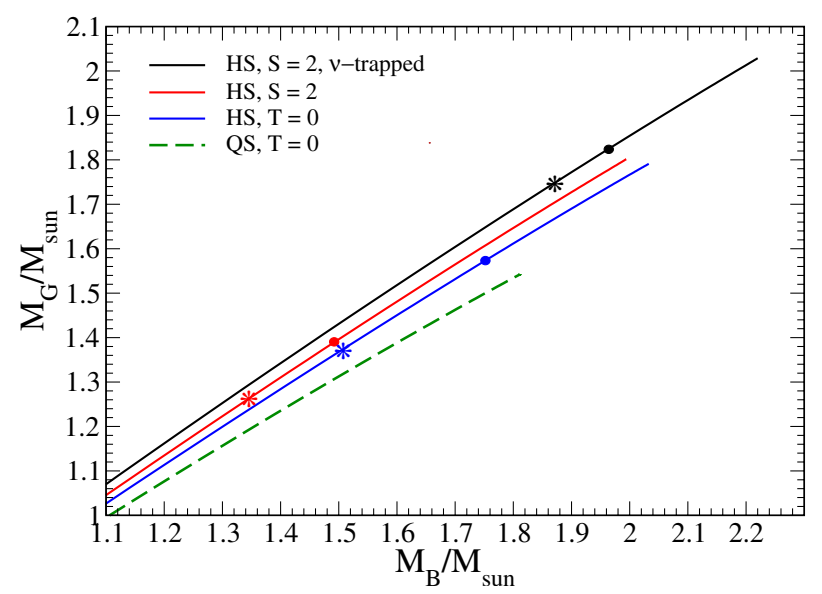

Fig. 11. (Color online) Same as Fig. 10, but with the additional stellar sequence (upper (black) line) for proto-hadronic stars with trapped neutrinos ( $v$ PHSs) with a lepton fraction $Y_{\mathrm{L}}=0.4$ and $\tilde{S}=2 k_{\mathrm{B}}$.

according to the results in Fig. 10, proto-hadronic stars with a baryonic mass $M_{B}<M_{B, \text { cr }}^{\mathrm{PHS}}\left(=1.492 M_{\text {sun }}\right.$ within the present EOS parametrzation) will survive $\mathrm{Q}^{*}$-matter early nucleation (i.e. nucleation within the cooling time $t_{\text {cool }} \sim$ a few $10^{2} \mathrm{~s}$ ) and in the end will form stable $(\tau=\infty)$ cold hadronic stars. Proto-hadronic stars with $M_{B, \mathrm{cr}}^{\mathrm{PHS}} \leq M_{B}<M_{B, \max }^{\mathrm{QS}}$ (the maximum baryonic mass of the cold QS sequence, $1.813 M_{\text {sun }}$ for the present EOS) will experience early nucleation of a $\mathrm{Q}^{*}$-matter drop and will ultimately form a cold deleptonized quark star. The last possibility is for PHSs having $M_{B}>M_{B \text {, max }}^{\mathrm{QS}}$. In this case the early nucleation of a $\mathrm{Q}^{*}$-matter drop will trigger a stellar conversion process to a cold QS configuation with $M_{B}>M_{B \text {, max }}^{\mathrm{QS}}$, thus these PHSs will finally form black holes.

The outcomes of this scenario are not altered by neutrino trapping effects in hot $\beta$-stable hadronic matter as illustrated in Fig. 11 where (in addition to the stellar sequence curves of Fig. 10) we plot the stellar sequence for proto-hadronic stars

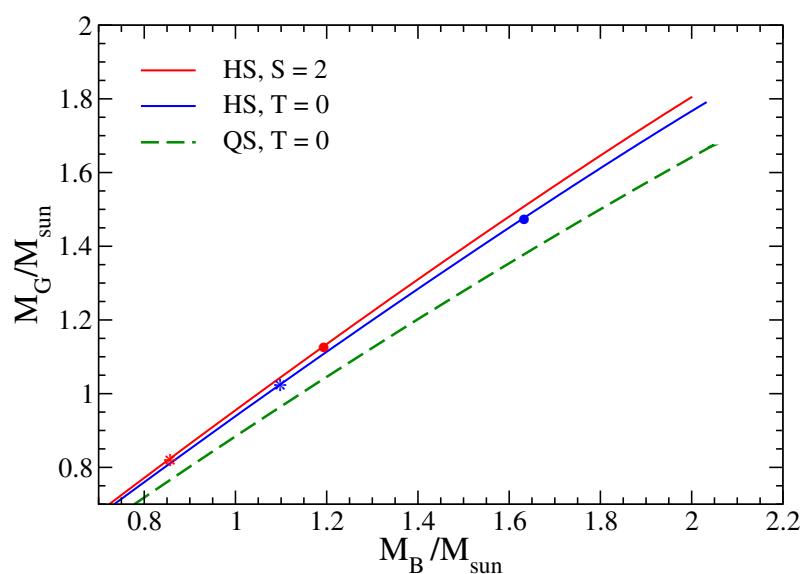

Fig. 12. (Color online) Same as Fig. 10, but with a bag constant $B=$ $70.9 \mathrm{MeV} / \mathrm{fm}^{3}$.

with trapped neutrinos ( $v$ PHSs) with a lepton fraction $Y_{\mathrm{L}}=0.4$ and $\tilde{S}=2 k_{\mathrm{B}}$. For the $v$ PHS stellar sequence in Fig. 11 we find a critical baryonic mass $M_{B, \mathrm{cr}}^{\nu \mathrm{PHS}}=1.96 M_{\text {sun }}$. Thus $v$ PHSs with $M_{B} \geq M_{B, \mathrm{cr}}^{\nu \mathrm{PHS}}$ after neutrino escape and cooling will finally evolve to black holes. The fate of a $v$ PHS with $M_{B}<M_{B, \mathrm{cr}}^{\nu \mathrm{PHS}}$ is the same as the corresponding neutrino-free PHS with equal baryonic mass.

Finally, in Fig. 12 we plot the PHS, cold HS, and cold QS sequences in the gravitational-baryonic mass plane for the case of a bag constant $B=70.9 \mathrm{MeV} / \mathrm{fm}^{3}$. (All the other EOS parameters are the same as for the results in Fig. 10) Apart from changes in the numerical values of the maximum masses of the various stellar sequences and of the values of the stellar critical masses, the evolutionary scenario of a proto-hadronic star is qualitatively similar to the one previously discussed for the results in Fig. 10.

An interesting situation comes up when a PHS with $M_{B}<$ $M_{B, \mathrm{cr}}^{\mathrm{PHS}}$ is formed in a binary stellar system. In this case, as we have just seen, a stable (case of Fig. 10) or a metastable (case of Fig. 12) cold HS is formed. Long-term accretion from the companion star will start to populate the portion of the cold HS sequence with $M_{B, \mathrm{cr}}^{\mathrm{PHS}}<M_{B}<M_{B \text {,cr. }}^{\mathrm{HS}}$. If the star accretes enough matter $\left(\sim 0.1-0.3 M_{\odot}\right.$, see Figs. 10 and 12$)$, it could possibly reach the critical mass $M_{B, \mathrm{HS}}^{\mathrm{HS}}$. At this point a quantum nucleation process will trigger the conversion of this critical mass cold HS to a QS (Berezhiani et al. 2002, 2003; Bombaci et al. 2004). The time delay between the supernova explosion forming the PHS, and the second "explosion" (neutrino burst or/and GRB) forming the QS will actually depend on the mass accretion rate, which modifies the quark matter nucleation time via an explicit time dependence of the stellar central pressure.

Within the present scenario, there is thus a stellar mass range $M_{B, \mathrm{cr}}^{\mathrm{PHS}}<M_{B}<M_{B, \mathrm{cr}}^{\mathrm{HS}}$ where it is possible to find two different types of "neutron stars" in the Universe: pure hadronic compact stars with large radii in the range $12-20 \mathrm{~km}$, and quark stars with small radii in the range of 6-9 km (Bombaci et al. 2004; Drago \& Lavagno 2010). Accurate measurements of both the mass and radius of a few individual "neutron stars" (Bhattacharyya 2010; Steiner et al. 2010) could shed light on the validity of this presumptive scenario.

\section{Limiting mass of compact stars}

The possibility of having metastable hadronic stars, together with the feasible existence of two distinct families of compact 
stars (pure hadronic stars and quark stars), demands an extension of the concept of maximum mass of a "neutron star" with respect to the classical one introduced by Oppenheimer \& Volkoff (1939). Since metastable HS with a "short" mean-life time are very unlikely to be observed, an extended concept of maximum mass has to be introduced in view of the comparison with the values of the mass of compact stars deduced from direct astrophysical observation. With this operational definition in mind, Bombaci et al. (2004) define the limiting mass $\left(M_{\mathrm{lim}}\right)$ of a compact star in the case of cold stellar configurations. This concept of limiting mass can be straightforwardly extended to the present case, i.e. taking the effects of proto-neutron stars evolution into account. For the two cases reported in Figs. 10 and 12, the limiting mass is the highest between the gravitational critical mass $M_{\mathrm{cr}}^{\mathrm{HS}}$ of the cold HS sequence and the gravitational maximum mass $M_{\mathrm{max}}^{\mathrm{QS}}$ of the cold QS sequence. Thus $M_{\mathrm{lim}}=M_{\mathrm{cr}}^{\mathrm{HS}}$ (case in Fig. 10) and $M_{\text {lim }}=M_{\max }^{\mathrm{QS}}$ (case in Fig. 12).

The very recent measurement (Demorest et al. 2010) of the Shapiro delay in the binary millisecond pulsar J1614-2230 has helped to obtain the mass of the associated neutron star. The calculated mass is $M=(1.97 \pm 0.04) M_{\odot}$ (Demorest et al. 2010), making PSR J1614-2230 the most massive neutron star known to date.

As is well known (see e.g. Lattimer \& Prakash 2001; Bombaci 2007), neutron star mass measurements give one of the most stringent tests of the composition and EOS of strong interacting matter at very high densities. Here, we very briefly address some possible implications of the high mass of PSR J1614-2230 in connection with the feasibility of the scenario discussed in present work. To this aim we consider two almost diametrical situations for the stiffness of the EOS.

The first possibility is for the case of a stiff hadronic EOS and a soft quark matter EOS. This is the case, for example, for the GM1 model with $x_{\sigma}=x_{\rho}=0.8, x_{\omega}=0.913$ for hyperonic matter, and the MIT bag model EOS (Farhi \& Jaffe 1984) with $B=100-150 \mathrm{MeV} / \mathrm{fm}^{3}$ for quark matter. For these EOS models, the limiting mass is given by the gravitational critical mass of the HS sequence, and it is in the range $M_{\lim }=1.9-2.1 M_{\odot}$ (Bombaci et al. 2008). Thus, in this case, PSR J1614-2230 can be interpreted as a pure hadronic star (hyperon star), and quark matter nucleation, in HS with $M>M_{\mathrm{cr}}^{\mathrm{HS}}$, will produce stellar configurations which will form black holes.

The second possibility is for a soft hadronic EOS and a stiff quark matter EOS. This is the case, for example, of the microscopic Brueckner-Hartree-Fock EOS for hyperonic matter (Baldo et al. 2000; Vidaña et al. 2000; Schulze et al. 2006) and the recent perturbative calculations of the quark matter EOS by (Kurkela et al. 2010) which include quark interaction effects up to the second order in the QCD coupling $\alpha_{\mathrm{s}}$ (see also Fraga et al. 2001; Alford et al. 2005). For these EOS models, the limiting mass is likely given by the gravitational maximum mass of the cold QS sequence, which according to the results of (Kurkela et al. 2010) is $M_{\max }^{\mathrm{QS}} \sim 2 M_{\odot}$ (hybrid stars), $M_{\max }^{\mathrm{QS}}=2.0-2.7 M_{\odot}$ (strange stars). Thus, for this second case, PSR J1614-2230 could be interpreted as a quark star (Özel et al. 2010).

\section{Conclusions}

In summary, in this work we have studied the quark deconfinement phase transition in hot $\beta$-stable hadronic matter and explored some of its consequences for the physics of neutron stars at birth. We calculated and compared the nucleation time due to thermal and quantum nucleation mechanisms, and computed the crossover temperature above which thermal nucleation dominates the finite temperature quantum nucleation mechanism. In addition, we introduced the new concept of limiting conversion temperature $\Theta$ for proto-hadronic stars and extended the concept of critical mass (Berezhiani et al. 2002, 2003; Bombaci et al. 2004) to the case of finite temperature hadronic stars.

Our main finding is that proto-hadronic stars with a gravitational mass lower than the critical mass $M_{\mathrm{cr}}$ could survive the early stages of their evolution without decaying to a quark star. This outcome contrasts with the predictions of the earlier studies (Horvath et al. 1992; Horvath 1994; Olesen \& Madsen 1994; Heiselberg 1995; Harko et al. 2004) where it was inferred that all the pure hadronic compact stars, with a central temperature above $2-3 \mathrm{MeV}$, are converted to quark stars within the first seconds after their birth.

However, the prompt formation of a critical size drop of quark matter could take place when $M>M_{\mathrm{cr}}$. These protohadronic stars evolve to cold and deleptonized quark stars or collapse to a black holes.

Finally, if quark matter nucleation occurs during the postbounce stage of core-collapse supernova, then the quark deconfinement phase transition could trigger a delayed supernova explosion characterized by a peculiar neutrino signal (Sagert et al. 2009; Mintz et al. 2010; Nakazato et al. 2008; Dasgupta et al. 2010).

Acknowledgements. This work has been partially supported by FCT (Portugal) under grants SFRH/BD/62353/2009, PTDC/FIS/64707/2006 and FCOMP-010124-FEDER-008393 with FCT reference CERN/FP/109316/2009, and by COMPSTAR, an ESF Research Networking Programme.

\section{References}

Aguilera, D. N., Blaschke, D., \& Grigorian, H. 2004, A\&A, 416, 991 Alford, M. G., Braby, M., Paris, M., \& Reddy, S. 2005, ApJ, 629, 969

Alford, M. G., Schmitt, A., Rajagopal, K., \& Schafer, T. 2008, Rev. Mod. Phys., 80,455

Baldo, M., Burgio, G. F., \& Schulze, H.-J. 2000, Phys. Rev. C, 61, 055801

Bambi, C., \& Drago, A. 2008, Astropart. Phys., 29, 223

Benhar, O., \& Valli, M. 2007, Phys. Rev. Lett., 99, 232501

Benhar, O., Polls, A., Valli, M., \& Vidaña, I. 2010, Phys. Rev. C, 81, 024305

Berezhiani, Z., Bombaci, I., Drago, A., Frontera, F., \& Lavagno, A. 2002, Nuclear Physics B - Proceedings Supplements, 113, 268

Berezhiani, Z., Bombaci, I., Drago, A., Frontera, F., \& Lavagno, A. 2003, ApJ, 586,1250

Bhattacharyya, S. 2010, Adv. Space Res., 45, 949

Bodmer, A. R. 1971, Phys. Rev. D, 4, 1601

Bombaci, I. 1996, A\&A, 305, 871

Bombaci, I. 2007, Eur. Phys. J. A, 31, 810

Bombaci, I., \& Datta, B. 2000, ApJ, 530, L69

Bombaci, I., Parenti, I., \& Vidaña, I. 2004, ApJ, 614, 314

Bombaci, I., Lugones, G., \& Vidaña, I. 2007, A\&A, 462, 1017

Bombaci, I., Panda, P. K., Providência, C., \& Vidaña, I. 2008, Phys. Rev. D, 77 083002

Bombaci, I., Logoteta, D., Panda, P. K., Providência, C., \& Vidaña, I. 2009, Phys. Lett. B, 680, 448

Burrows, A., \& Lattimer, J. M. 1986, ApJ, 307, 178

Casalbuoni, R., \& Nardulli, G. 2004, Rev. Mod. Phys., 76, 263

Chattaerjee, D., \& Bandyopadhyay, D. 2006, Phys. Rev. D, 74, 023003

Csernai, L., \& Kapusta, J. I. 1992, Phys. Rev. D, 46, 1379

Danielewicz, P. 1984, Phys. Lett. B, 146, 168

Dasgupta, B., Fischer, T., Horiuchi, S., Liebendörfer, M., \& Mirizzi, A. 2010, Phys. Rev. D, 81, 103005

Demorest, P. B., Pennucci, T., Ransom, S. M., Roberts, M. S. E., \& Hessel, J. W. T. 2010, Nature, 467, 1081

Drago, A., \& Lavagno, A. 2010, [arXiv: 1004.0325$]$

Drago, A., Lavagno, A., \& Pagliara, G. 2004, Phys. Rev. D, 69, 057505

Drago, A., Lavagno, A., \& Parenti, I. 2007, ApJ, 659, 1519

Drago, A., Pagliara, G., \& Schaffner-Bielich, J. 2008, J. Phys. G, 35, 014052

Farhi, E., \& Jaffe, R. L. 1984, Phys. Rev. D, 30, 2379 
Fodor, Z., \& Katz, S. D. 2004, Prog. Theor. Suppl., 153, 86

Flowers, E., \& Itho, N. 1979, ApJ, 230, 847

Fraga, E. S., Pisarski, R. D., \& Schaffner-Bielich, J. 2001, Phys. Rev. D, 63, 121702(R)

Glendenning, N. K. 2000, Compact Stars: Nuclear Physics, Particle Physics, and General Relativity (New York: Springer)

Glendenning, N. K., \& Moszkowski, S. 1991, Phys. Rev. Lett., 67, 2414

Gu, J.-F., Guo, H., Lee, X.-G, Liu Y.-X., \& Xu, F.-R. 2008, Commun. Theor. Phys., Beijing, China, 49, 461

Harko, T., Cheng, K. S., \& Tang, P. S. 2004, ApJ, 608, 945

Heiselberg, H., Baym, G., \& Pethick, C. J. 1991, Nucl. Phys. B, Proc. Suppl., 24,144

Heiselberg, H., Pethick, C. J., \& Staubo, E. F. 1993, Phys. Rev. Lett., 70, 1355

Heiselberg, H. 1995, in Strangeness and Quark Matter, ed. G. Vassiliadis (World Scientific), 338, [arXiv:hep-ph/9501374]

Hidaka, Y., McLerran, L., \& Pisarski, R. D. 2008, Nucl. Phys. A, 808, 117

Horvath, J. E., Benvenuto, O. G., \& Vucetich, H. 1992, Phys. Rev. D, 45, 3865

Horvath, J. E. 1994, Phys. Rev. D, 49, 5590

Hsu, S. D. H., \& Schwetz, M. 1998, Phys. Lett. B, 432, 2003

Iida, K., \& Sato, K. 1997, Prog. Theor. Phys., 98, 277

Iida, K., \& Sato, K. 1998, Phys. Rev. C, 58, 2538

Karsch, F. 2005, J. Phys. G, 31, S633

Kurkela, A., Romatschke, P., \& Vuorinen, A. 2010, Phys. Rev. D, 81, 105021

Langer, J. S. 1968, Phys. Rev. Lett., 21, 973

Langer, J. S. 1969, Ann. Phys. (N.Y.), 54, 258

Langer, J. S., \& Turski, L. A. 1973, Phys. Rev. A, 8, 3230

Lattimer, J. M., \& Prakash, M. 2001, ApJ, 550, 426

Lifshitz, I. M., \& Kagan, Y. 1972, Sov. Phys. JETP, 35, 206

Lombardo, M. P. 2007, Mod. Phys. Lett., A, 22, 457

Lugones, G., \& Benvenuto, O. G. 1998, Phys. Rev. D, 58, 083001

Lugones, G., \& Bombaci, I. 2005, Phys. Rev. D, 72, 065021

Lugones, G., Grunferld, A. G., Scoccola N. N., \& Villavicencio, C. 2009, Phys. Rev. D, 80, 045017
Lugones, G., do Carmo, T. A. S., Grunferld, A. G., \& Scoccola, N. N. 2010, Phys. Rev. D, 81, 085012

McLerran, L., \& Pisarski, R. D. 2007, Nucl. Phys. A, 796, 83

Menezes, D. P., \& Providência, C. 2003, Phys. Rev. C, 68, 035804

Mintz, B. W., Fraga, E. S. Pagliara, G., \& Schaffner-Bielich, J. 2010, Phys. Rev. $\mathrm{D}, 81,123012$

Nakazato, K., Sumiyoshi, K., \& Yamada, S. 2008, Phys. Rev. D, 77, 103006

Olesen, M. L., \& Madsen, J. 1994, Phys. Rev. D, 49, 2698

Olinto, A. V. 1987, Phys. Lett. B, 192, 71

Oppenheimer, J. R., \& Volkoff, G. M. 1939, Phys. Rev., 55, 374

Özel, F., Psaltis, D., Ransom, S., Demorest, P., \& Alford, M. 2010, ApJ, 724, L199

Prakash, M., Bombaci, I., Prakash, M., et al. 1997, Phys. Rep., 280, 1

Pons, J. A., Reddy, S., Prakash, M., Lattimer, J. M., \& Miralles, J. A. 1999, ApJ, 513,780

Sagert, I., Fischer, T., Hempel, M., et al. 2009, Phys. Rev. Lett., 102, 081101

Sandin, F., \& Blaschke, D. 2007, Phys. Rev. D, 75, 125013

Schulze, H.-J., Polls, A., Ramos, A., \& Vidaña, I. 2006, Phys. Rev. C, 73, 058801

Sedrakian, A. D., Blaschke, D., Röpke, G., \& Schultz, H. 1994, Phys. Lett. B, 338,111

Steiner, A. W., Lattimer, J. M., \& Brown, E. F. 2010, ApJ, 722, 33

Serot, B. D., \& Walecka, J. D. 1986, Adv. Nucl. Phys., 16, 1

Turski, L. A., \& Langer, J. S. 1980, Phys. Rev. A, 22, 2189

van Dalen, E. N. E., \& Dieperink, A. E. L. 2004, Phys. Rev. C, 69, 025802

Venugopalan, R., \& Vischer, A. P. 1994, Phys. Rev. E, 49, 5849

Vidaña, I., Polls, A., Ramos, A., Engvik, L., \& Hjorth-Jensen, M. 2000, Phys. Rev. C, 62, 035801

Vidaña, I., Bombaci, I., \& Parenti, I. 2005, J. Phys. G, 31, S1165

Walecka, J. D. 1974, Ann. Phys. (N.Y.), 83, 491

Witten, E. 1984, Phys. Rev. D, 30, 272

Shternin, P. S., \& Yakovlev, D. G. 2008, Phys. Rev. D, 78, 063006

Zhang, H. F., Lombardo, U., \& Zuo, W. 2010, Phys. Rev. C, 82, 015805 